\title{
Characterization of liver Disease Based on Ultrasound Imaging System
}

\author{
Mohammed K. Bin jaah, Abdullah Aljuhani, Umar S. Alqasemi
}

\begin{abstract}
Computer-Aided Detection (CAD) systems are one of the most effected tools nowadays in aiding physicians in the detection of liver tumors at early stage. In this paper, the CADe system will be built which has the ability to detect the abnormal tumor inside the liver. In order to create that system, different types of classifiers must be implemented. In our CADe system, a support vector machine (SVM) and K-Nearest Neighbor (KNN) will be used as classifiers. A total number of 120 images including the normal and abnormal cases were collected. Initially, the features will be extracted from database images in order to distinguish between the classes of those liver tumors. Then, by using SVM and KNN the images will be classified into two classes normal and abnormal cases. The paper reveals that SVM and KNN, which demonstrated 100 percent precision, 100 percent sensitivity, and 100 percent specificity, were the best classifiers.

Keywords : Computer aided detection system, Liver cancer, Classification, SVM, KNN.
\end{abstract}

\section{INTRODUCTION}

In the human body, the liver is a very important organ which plays a major role in getting rid of dangerous substances that could seriously affect the body. Too many diseases that may mess with its function one of the common diseases is livers tumors. Livers tumors are irregular masses of tissue that develop as the cells begin to replicate at an elevated rate. The cause of this is either inherited (genetic) or caused by a variety of factors such as viruses, drug usage or obesity. Liver tumors can be noncancerous and cancerous [1]. In the United States, approximately 24,000 men and 10,000 women got liver cancer annually (about 18,000 men and 9,000 women who die because of this disease). Last year, the number of people who have liver cancer increased [2]. Elsewhere, there are around 6,100 new cases of liver cancer per year in the UK. Liver cancer is one of the most common forms of cancer, and accounts for $2 \%$ of all new cases of cancer. In Great Britain, liver cancer is the largest age between 75 to 89 , where $43 \%$ of all new patients diagnosed with liver cancer each year are 75 or older[3]. For cancerous liver tumors, there are two-type primary liver cancer, which is located in the liver. The second type is Metastatic liver cancer, which spread from other cancer sites in the body. There are so many symptoms in order

Manuscript received on January 15, 2021.

Revised Manuscript received on February 02, 2021.

Manuscript published on February 28, 2021

Mohammed K. Binjaah*, Department of Biomedical Engineering, King Abdul-Aziz University, KSA.

Abdullah Aljuhani, Biomedical Engineer, Ministry of Health, KSA and Studying MSc Electrical and Biomedical Engineering, King Abdulaziz University, KSA.

Umar Alqasemi, Associate Professor, Department of Electrical and Computer Engineering, King Abdulaziz University, KSA.

(c) The Authors. Published by Blue Eyes Intelligence Engineering and Sciences Publication (BEIESP). This is an open access article under the CC BY-NC-ND license (http://creativecommons.org/licenses/by-nc-nd/4.0/) to determine liver diseases. The most common symptoms are abdominal pain and swelling, yellowish skin and eyes, itchy skin, swelling of the legs and feet, pale stool color, black urine color, chronic weakness, nausea or vomiting, lack of appetite, propensity to bruise easily [4]. The focus of this paper research is developing a computer-Aided detection (CAD) system using MATLAB tools to diagnose liver tumor in the ultrasound imaging. This new methodology would help the medical professionals to indict liver tumors fast at early stage; therefore, they can make the right decision in the treatment. The built CAD system shall have the ability to detect the abnormal activities inside the scanned liver images. In creating such CAD system different type of classifiers are implemented. These classifiers are support vector machine (SVM); which is a machine learning algorithm that can used for classification or regression between different datasets and it mostly used for nonlinear dataset using hyperplane. Other classifier used is K-Nearest Neighbor (KNN); which is a machine learning algorithm that can used for classification based on the nearest $\mathrm{K}$ point [5]. In this study, the ultrasound liver tumor imaging will be analyzed using MTLAB tool. Medical ultrasound of liver tumor included two levels which are detection and characterization. Tumor detection is depended on the action of the method and must include morphometric information and topographic information. The characterization of these data is necessary for the presentation of liver tumors and diagnosis. Tumor characterization is a complicated method based on a sum of criteria driving towards tumor nature information. Often, other diagnostic methods, particularly interventional ones are no longer needed [6].

\section{LITERATURE REVIEW}

Sendren Sheng-Dong Xu, Chun-Chao Chang, Chien-Tien Su, and Pham Quoc Phu [7] explored the use of computer-aided diagnosis (CAD) to discriminate between hepatocellular carcinoma (HCC), the most common form of primary liver malignancy that causes death in people with cirrhosis worldwide, and liver abscess, based on the characteristics of the ultrasound image texture and the description of the support vector machine (SVM). They derived 52 gray-level co-occurrence matrix (GLCM) features and 44 gray-level run-length matrix (GLRLM) features from the ultrasound picture regions of interest (ROIs). Their proven method classifies liver cancer and liver abscess with an accuracy of 88.875 percent by SVM. Yoo Na Hwanga, Ju Hwan Leeb, Ga Young Kimb, Yuan Yuan Jiangb, and Sung Min Kim [8] in their paper, they focused on the improvement of the diagnostic efficacy of focal liver lesions by quantifying the main characteristics of ultrasound images of hemangiomas, cysts, and malignant lesions.

Published By:

Blue Eyes Intelligence Engineering and Sciences Publication

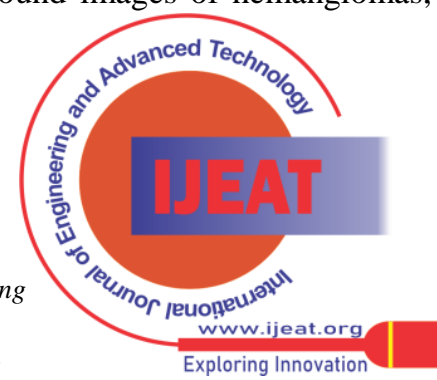




\section{Characterization of liver Disease Based on Ultrasound Imaging System}

The main component analysis (PCA) used to construct a collection of inputs for a nerve network was chosen to have a total of 29 core features. According to the three classes, the accuracy is compared, and different feature sets are calculated by running a PCA to test each group's classification accuracy. The accuracy was 97.72 percent between a cyst and a hemangioma when the means is chosen as the optimum feature group and 97.63 percent between a cyst and a malignant lesion. Compared to the mean the diagnostic precision of autocorrelation improved significantly by $22,14 \%$, to differentiate between a malignant lesion and the hemangioma. Contrast nevertheless contributed to an increase in accuracy of $18.44 \%$ due to the classification of hemangioma and a malignant lesion, but did not produce a substantial difference in autocorrelation accuracy where a cyst and a hemangioma were identified or between a cyst and a malignant lesion. Contrast demonstrated high diagnostic accuracy in all classification classes of more than 90 percent. D Santhosh Reddy, R Bharath and P Rajalakshmi [9] explained how to establish the classification precautions for the Fatty Liver Disease, (FLD) depend on ultrasound images, by using computer-aided diagnostic techniques. Their success review explains that $90.6 \%$ accuracy in classifying ordinary and fatty liver pictures is supported by the proposed system.

Andreia Andrade1, José Silvestre Silva, Jaime Santos4, Pedro Belo-Soares [10] demonstrated a semiautomatic classification approach to test statistic liver tissues by using B-scan ultrasound images. Different functions, such as Artificial Neural Networks (ANN), Support Vector Machines (SVM), and k-nearest neighbors (KNN), were extracted and used in three different classifications. They experimented on 325 features that collected liver ultrasound images from each ROI, namely 10 using FOS, 44 using GLRLM, 198 using GLCM, 70 using TEM, and 3 using the method of Fractals. Their ANN classifiers resulted in 76.92 percent precision, 79.77 percent precision resulted in SVM classifiers, and 74.05 percent precision resulted in SVM classifiers. M. B. Subramanya, Vinod Kumar, Shaktidev Mukherjee \& Manju Saini [11] introduced the diagnostic ranks of fatty hepatic illnesses (i.e. moderate, medium, and extreme fatty liver along with normal liver tissue) which added by using the Computer-Aided Diagnose (CAD) method. Ultrasound images composed of 12 regulars, 14 medium, 14 moderateand 13 extreme liver fat images are used in fifty-three B-mode. A differential evolution feature selection (DEFS) algorithm obtained the sub-sets of optimal features and a support vector machine (SVM) was used for the classification task. They obtained an average precision and standard deviation of $81 \pm 3.3$ and 83.5 \pm 3.3 after feature selection. Yu Masuda , Tomoko Tateyama , Wei Xiong, and Jiayin Zhou [12] discuss the automatic detection of the liver tumors using CT images. In the paper, they propose a new method for the automatic detection of the liver tumors base on the estimation normal density of the liver shows in CT images. Then, the expectation maximization of the posterior marginal algorithm utilized to detect tumorous regions. Lastly, the noise reduced by applying a shape constraint in order to identify focal tumors. The results show it is accuracy and effectively in detecting tumors even in poor-contrast CT images. V.Ulagamuthalvi, D.Sridharan [13] examined the automated recognition of the ultrasound liver tumor image. Where the proposed method is approached by the following. First of all, by measuring the textural feature from the co-occurrence matrix and the run length process, they segment the liver image. Next, a general algorithm based on risk constrained statistical learning theory is used for the classification support vector machine (SVM), such as optical character recognition. Then, the textural features are given separately for the study train and test datasets as input to the SVM for various function methods. Finally, when the SVM classifier device is given an ultrasound liver tumor image, the characteristics are determined, and then either a normal liver image or an abnormal image is known.

Wassem Abdulrahman [14], they developed an automatic means of separating abnormal regions of the liver area into the abdominal area in CT images by using a computer-aided diagnostic (CAD) device. The approach relies on the use of the counting algorithm for linked compounds (CCL) and the use of the FCM algorithm for data collection. The findings show that the automatic extraction process for the liver region has been successfully developed; however, extraction and detection of the liver tumor was not effective and requires improvements.

Table 1. Summary of previous researches results

\begin{tabular}{|c|c|c|c|c|}
\hline $\begin{array}{c}\text { References } \\
\text { No. }\end{array}$ & [7] & [8] & [9] & [10] \\
\hline Features used & $\begin{array}{c}\text { (GLCM) and } \\
\text { (GLRLM) } \\
\text { from (ROIs) }\end{array}$ & $\begin{array}{c}\text { First order } \\
\text { statistic (FOS) } \\
\text { and (GLRLM) }\end{array}$ & $\begin{array}{c}\text { Fatty liver } \\
\text { Disease (FLD) } \\
\text { in ultrasound } \\
\text { images }\end{array}$ & $\begin{array}{c}\text { GLCM and } \\
\text { GLRLM from } \\
\text { ROI }\end{array}$ \\
\hline $\begin{array}{c}\text { Classifiers } \\
\text { used }\end{array}$ & SVM & $\begin{array}{c}\text { And artificial } \\
\text { neural } \\
\text { network } \\
\text { (ANN) }\end{array}$ & $\begin{array}{c}\text { Convolution } \\
\text { neural network } \\
\text { (CNN) }\end{array}$ & $\begin{array}{c}\text { ANN, SVM } \\
\text { and KNN }\end{array}$ \\
\hline Accuracy & $88.875 \%$ & $93.13 \%$ & $90.6 \%$ & $\begin{array}{c}\text { ANN 76.92\% } \\
\text { SVM79.77\% } \\
\text { KNN 74.05\% }\end{array}$ \\
\hline Sensitivity & NP & NP & $95 \%$ & NP \\
\hline Specificity & NP & NP & $85 \%$ & NP \\
\hline
\end{tabular}

\begin{tabular}{|c|c|c|c|c|}
\hline References No. & [11] & [12] & [13] & [14] \\
\hline Features used & $\begin{array}{c}\text { Fatty liver } \\
\text { disease using } \\
\text { DEFS which is } \\
\text { FOS and } \\
\text { GLCM }\end{array}$ & $\begin{array}{c}\text { Automatic } \\
\text { detection of } \\
\text { the liver } \\
\text { tumors base } \\
\text { on the } \\
\text { estimation } \\
\text { normal } \\
\text { density of } \\
\text { the liver } \\
\text { shows in CT } \\
\text { images }\end{array}$ & $\begin{array}{c}\text { Co-occurrenc } \\
\text { e matrix and } \\
\text { run length } \\
\text { method }\end{array}$ & $\begin{array}{c}\text { Compounds } \\
\text { numbering } \\
\text { algorithm } \\
\text { CCL and FCM } \\
\text { Algorithm }\end{array}$ \\
\hline Classifiers used & SVM & $\begin{array}{c}\text { (EM/ MPM) } \\
\text { algorithm }\end{array}$ & SVM & KNN \\
\hline Accuracy & $81 \%$ & $94 \%$ & $96.72 \%$ & $86 \%$ \\
\hline Sensitivity & $\mathrm{NP}$ & $\mathrm{NP}$ & $\mathrm{NP}$ & $\mathrm{NP}$ \\
\hline Specificity & $\mathrm{NP}$ & $\mathrm{NP}$ & $\mathrm{NP}$ & $\mathrm{NP}$ \\
\hline
\end{tabular}

\section{METHODOLOGY}

In this research paper, data of 120 liver images were analyzed using MATLAB. The analysis took place in five steps in order to discern between the normal and abnormal liver. These steps are as showing in the following chart:

Published By:

Blue Eyes Intelligence Engineering and Sciences Publication

(C) Copyright: All rights reserved.

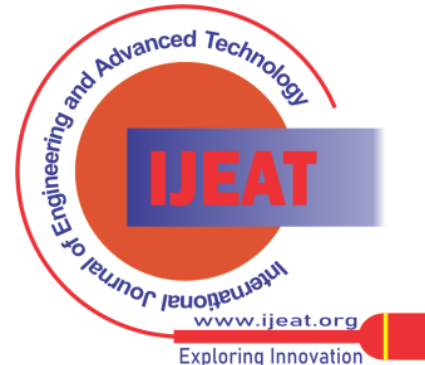




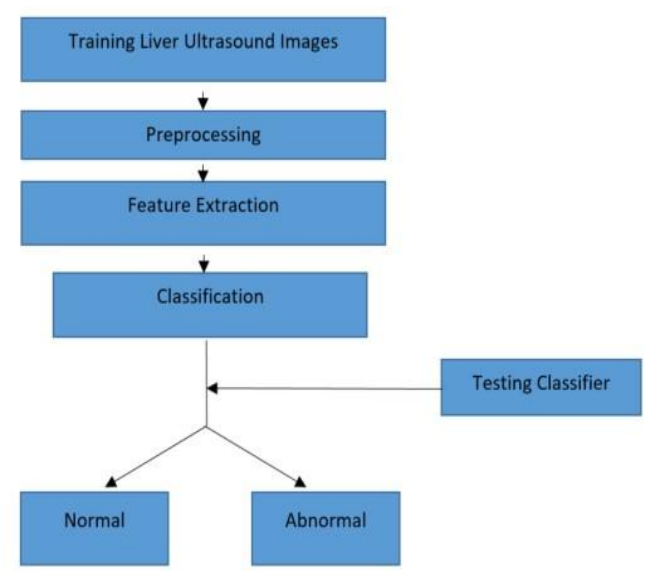

Figure 1. Flowchart of Proposed CAD System for liver tumor

\subsection{DATABASE SOURCE:}

University of Oxford database were used through this research study [15]. The database includes 227 cross-sectional images, of which 29 mice were collected with $1 \mathrm{~mm}$ step-by-step movement of the screen mounted on a manual positioning system with a resolution of 289 x 648 pixels, of hind leg xenograft tumors. Photos of the liver tumor were diagnosed using a $10 \mathrm{MHz}$ linear transducer ultrasound device and $50 \mathrm{MHz}$ screening. In this study, 120 images (42 normal and 42 abnormal) and 36 images used to test the performance of the classifiers. For research purposes only, the dataset is open and accessible.

\subsection{FEATURE EXTRACTION:}

In this section, feature selection will be discussed which plays an essential role in distinguishing between normal and abnormal cases. First of all, we computed 25 features beginning with the first-order statistics (21) and texture features (4 GLCM features). Then, we selected 19 features that have a p-value $<5 \%$ and give a high accuracy in the testing section. The statistical features involving mean, median, max, min, standard deviation, kurtosis, var and quantile $(0.1,0.2,0.3,0.4)$ are the most beneficial features used in this study after several times of trial, those features offer the best result of t-test significance along with the best classification output. The classification stage is the next step which depends on the category of images used in the extraction of features.

\subsection{CLASSIFICATION}

In previous stages, we collected the database and selected the features that gave us the desired results. In the classification stage, we divided the whole images that include the normal and abnormal into two main phases, learning and testing phases. In the learning phase, the collected data describe sharply the nature of the tumor if it normal or abnormal in order to guide and train the classifier. In the testing phase, by using the trained structure of the classifier the classification was accomplished. The selected features were used with 8 classifiers (Three SVM classifiers of the different kernel (Linear, Polynomial, and Radial Basis Function) and level 1,2,3,4, and 5 K-voting Nearest Neighbor (KNN) classifiers.

\section{RESULTS}

After running the MATLAB code and testing the train set of the images, the T-Test has a number of useful features $(\mathrm{P}$-Value $<0.05)=19$ out of 19. This means that all statistical features used are valuable and may offer good performance in

Published By:

Blue Eyes Intelligence Engineering and Sciences Publication table below: in some classifiers. studies.

\begin{tabular}{|c|c|c|c|c|}
\hline \multicolumn{7}{|c|}{ Table 2. Results of the classifiers } \\
\hline Indices (\%) & SVM rbf & SVM Poly & SVM Linear & KNN 1 \\
\hline Sensitivity & $100 \%$ & $50 \%$ & $50 \%$ & $100 \%$ \\
\hline Specificity & $100 \%$ & NaN & NaN & $100 \%$ \\
\hline PPV & $100 \%$ & $100 \%$ & $100 \%$ & $100 \%$ \\
\hline NPV & $100 \%$ & $0 \%$ & $0 \%$ & $100 \%$ \\
\hline Accuracy & $100 \%$ & $50 \%$ & $50 \%$ & $100 \%$ \\
& & & & \\
\hline Error & $0 \%$ & $50 \%$ & $50 \%$ & $0 \%$ \\
& & & & \\
\hline
\end{tabular}

Table 2 indicates that the best classifiers were SVMrbf, KNN1 and KNN2 with the same accuracy $=100 \%$ and Sensitivity $=100 \%$. Comparing with some studies as shown in table 3, as the applied order was a combination of the first and high order of the features, the final results of our study were valuable and gave a high accuracy, sensitivity, and specificity

Table 3. Comparing our best results of SVM and KNN with other

\begin{tabular}{|c|c|c|c|c|}
\hline $\begin{array}{c}\text { Indices } \\
\text { (\%) }\end{array}$ & $\begin{array}{c}\text { KNN } \\
\mathbf{2}\end{array}$ & KNN3 & KNN 4 & KNN 5 \\
\hline Sensitivity & $100 \%$ & $100 \%$ & $100 \%$ & $100 \%$ \\
\hline Specificity & $100 \%$ & $64.52 \%$ & $64.52 \%$ & $64.52 \%$ \\
\hline PPV & $100 \%$ & $45 \%$ & $45 \%$ & $45 \%$ \\
\hline NPV & $100 \%$ & $100 \%$ & $100 \%$ & $100 \%$ \\
\hline Accuracy & $100 \%$ & $72.50 \%$ & $72.50 \%$ & $72.50 \%$ \\
\hline Error & $0 \%$ & $27.50 \%$ & $27.50 \%$ & $27.50 \%$ \\
& & & & \\
\hline
\end{tabular}

\begin{tabular}{|c|c|c|c|c|}
\hline $\begin{array}{c}\text { References } \\
\text { No. }\end{array}$ & $\begin{array}{c}\text { Classifiers } \\
\text { used }\end{array}$ & Accuracy & Sensitivity & Specificity \\
\hline $\mathbf{7}$ & SVM & $88.88 \%$ & NP & NP \\
\hline $\mathbf{8}$ & SVM & $93.13 \%$ & NP & NP \\
\hline $\mathbf{1 0}$ & SVM & $79.77 \%$ & NP & NP \\
\cline { 2 - 5 } & KNN & $74.05 \%$ & NP & NP \\
\hline $\mathbf{1 1}$ & SVM & $81 \%$ & NP & NP \\
\hline $\mathbf{1 3}$ & SVM & $96.72 \%$ & NP & NP \\
\hline $\mathbf{1 4}$ & KNN & $86 \%$ & NP & NP \\
\hline Our study & SVM & $100 \%$ & $100 \%$ & $100 \%$ \\
\cline { 2 - 5 } & KNN & $100 \%$ & $100 \%$ & $100 \%$ \\
\hline
\end{tabular}

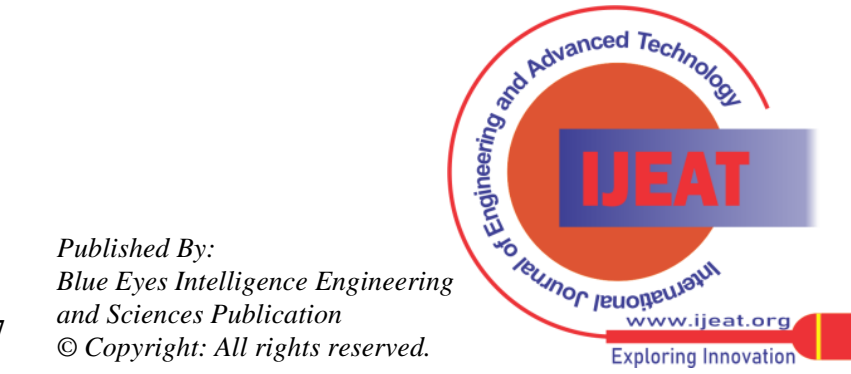
Exploring Innovation 


\section{Characterization of liver Disease Based on Ultrasound Imaging System}

\section{CONCLUSION \& DISSCUSSION}

In this research paper, a computer-Aided Detection (CAD) system using MATLAB tools was developed to help radiologists in early detection of abnormalities in ultrasound images of the liver. Eight classifiers were analyzed using 120 images (60 normal \& 60 abnormal); where the best results came up from SVMrbf ,KNN1 and KNN2 classifier with 100 $\%$ of accuracy \& sensitivity and $0 \%$ of error as shown in Table 2. University of Oxford database were used through this research study which includes 227 cross-sectional images. The results were obtained and found in this study is very good comparing with other developed study for computer-Aided detection (CAD) system that was found in the literature review section. Lastly, it is recommended in the future to use more liver tumor images from different database in order trained the classifier and test its performance.

\section{REFERENCES}

1. Liver disease. (2020, February 21). Retrieved December 15, 2020, from

https://www.mayoclinic.org/diseases-conditions/liver-problems/symp toms-causes/syc-20374502.

2. [Online] Centers For Disease Dontrol And Prevention, December 1 2020, Available: https://www.cdc.gov/cancer/liver/index.htm.

3. [Online] Cancer Research UK, 2018, Available: https://www.cancerresearchuk.org/health-professional/cancer-statistic s/statistics-by-cancer-type/liver-cancer\#heading-Zero.

4. Benjamin Wedro, M. (2019, November 07). Liver Disease Symptoms, Signs, Diet \& Treatment. Retrieved December 15, 2020, from https://www.medicinenet.com/liver_disease/article.htm

5. Giuseppe Ciaburro, MATLAB for Machine Learning August 2017.

6. Hironori Tanaka. Current role of ultrasound in the diagnosis of hepatocellular carcinoma 13 March 2020.

7. Sendren Sheng-Dong Xu, Chun-Chao Chang, Chien-Tien Su, Pham Quoc Phu, Tifany Inne Halim, and Shun-Feng Su. Classification of Hepatocellular Carcinoma and Liver Abscess by Applying Neural Network to Ultrasound Images, researchgate 20 Aug. 2020.

8. Yoo Na Hwanga, Ju Hwan Leeb, Ga Young Kimb, Yuan Yuan Jiangb, and Sung Min Kim. Classification of focal liver lesions onultrasound images by extracting hybrid textural features and using an artificial neural network, Bio-Medical Materials and Engineering 26 (2015) S1599-S1611.

9. D Santhosh Reddy, $\mathrm{R}$ Bharath and $\mathrm{P}$ Rajalakshmi, A Novel Computer-Aided Diagnosis Framework Using Deep Learning for Classification of Fatty Liver Disease in Ultrasound Imaging. The Institute of Electrical and Electronics Engineers (IEEE) 17-20 Sept. 2018.

10. Andreia Andrade1, José Silvestre Silva, Jaime Santos4, Pedro Belo-Soares, Classifier Approaches for Liver Steatosis using Ultrasound Images. Procedia technology 5 (2012). 763-770.

11. M. B. Subramanya, Vinod Kumar, Shaktidev Mukherjee \& Manju Saini, SVM-Based CAC System for B-Mode Kidney Ultrasound Images. Journal of digital imaging 28 Aug. 2015.

12. Masuda, Y., \& Tateyama, T. (2012, February). Liver Tumor Detection in CT Images by Adaptive Contrast Enhancement and the EM/MPM Algorithm. Retrieved December 15, 2020, from https://www.researchgate.net/publication/221923839_Liver_Tumor_ Detection_in_CT_Images_by_Adaptive_Contrast_Enhancement_and _the_EMMPM_Algorithm

13. Ulagamuthalvi, V., \& Sridharan, D. (2012, March 25). Automatic Identification of Ultrasound Liver Cancer Tumor Using Support Vector Machine. Retrieved December 15, 2020, from http://citeseerx.ist.psu.edu/viewdoc/download?doi=10.1.1.1081.3599 \&rep=rep1\&type=pdf

14. Abdulrahman, W. (2014, April 4). Diagnosis of Liver Tumors Using Image Processing. Retrieved December 15, 2020, from https://www.ijert.org/research/diagnosis-of-liver-tumors-using-imageprocessing-IJERTV3IS042128.pdf.

15. [Online] Institute of Biomedical Engineering - University of Oxford Available: http://omar.alkadi.net/797-2/.

\section{AUTHORS PROFILE}

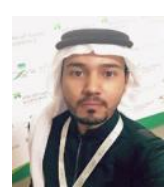

Mohammed K. Binjaah, Received bachelor degree in Biomedical Engineering from King Abdul-Aziz University, KSA. Currently studying MSc. Electrical and Biomedical Engineeringin King Abul-Aziz University, KSA.

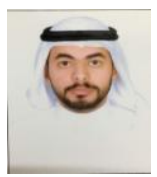

Abdullah Aljuhani, Received Bachelor degree in biomedical engineering from Purdue University, USA .Currently, Working as Biomedical Engineer in Ministry of Health, KSA and Studying MSc electrical and biomedical engineering at King Abdulaziz University, KSA.

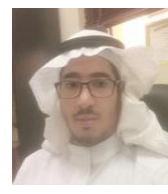

Dr. Umar S. Alqasemi, Associate Professor of Biomedical Engineering at the Dept. of Electrical and Computer Engineering, King, Abdulaziz University, Jeddah 21589, Saudi Arabia. PhD and MSc degree in Biomedical Engineering from UConn, USA. Research work in ultrasound, optical and image processing.

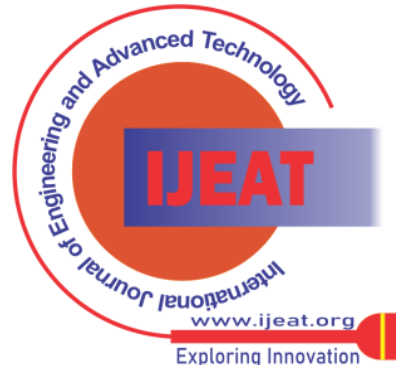

